\title{
Standardized Measurement Error as a Universal Measure of Data Quality for Event-Related Potentials: An Overview
}

Steven J. Luck ${ }^{1,2}$, Andrew X. Stewart ${ }^{1}$, Aaron Matthew Simmons ${ }^{1,2}$, and Mijke Rhemtulla ${ }^{2}$

Center for Mind \& Brain ${ }^{1}$ and Department of Psychology ${ }^{1,2}$, University of California, Davis

This manuscript was made possible by a grant from the National Institute of Mental Health (grant R01MH087450 to S.J.L.)

\begin{abstract}
Although it is widely understood that event-related potentials (ERPs) can be very noisy, the field has no widely accepted metric of ERP data quality. Here we propose a universal measure of data quality for ERP research, the standardized measurement error (SME). The SME is a simple extension of the widely used standard error of measurement, but it quantifies the error of single-participant measurements rather than group values. In addition, the SME quantifies data quality with respect to the specific amplitude or latency value being measured in a given study (e.g., the peak latency of the P3 wave). The SME is trivial to compute during the averaging process for linear measures such as mean amplitude, and it can be extended to virtually any amplitude or latency measure via a straightforward bootstrapping approach.
\end{abstract}




\section{INTRODUCTION}

By averaging together multiple single-trial EEG epochs, we can obtain averaged ERP waveforms that differ consistently across groups or conditions. In many experiments, however, we cannot obtain enough trials to obtain clean averaged ERP waveforms, and the residual noise in the data can dramatically distort our data and reduce our ability to find significant differences. Moreover, ERP data quality can vary widely across studies as a result of differences in the number of trials being averaged together, the quality of EEG recordings, and the analysis procedures. However, the field has not adopted a standard metric of data quality that can be used to quantify the noise level in a given study or determine which recording and analysis procedures lead to the best data quality.

One reason for this is that existing metrics - such as the root mean square of the voltage in the prestimulus period (Luck, 2014) - do not necessarily capture the noise that is relevant for a given experiment. As an example, Figure 1 shows how high-frequency noise can distort the peak
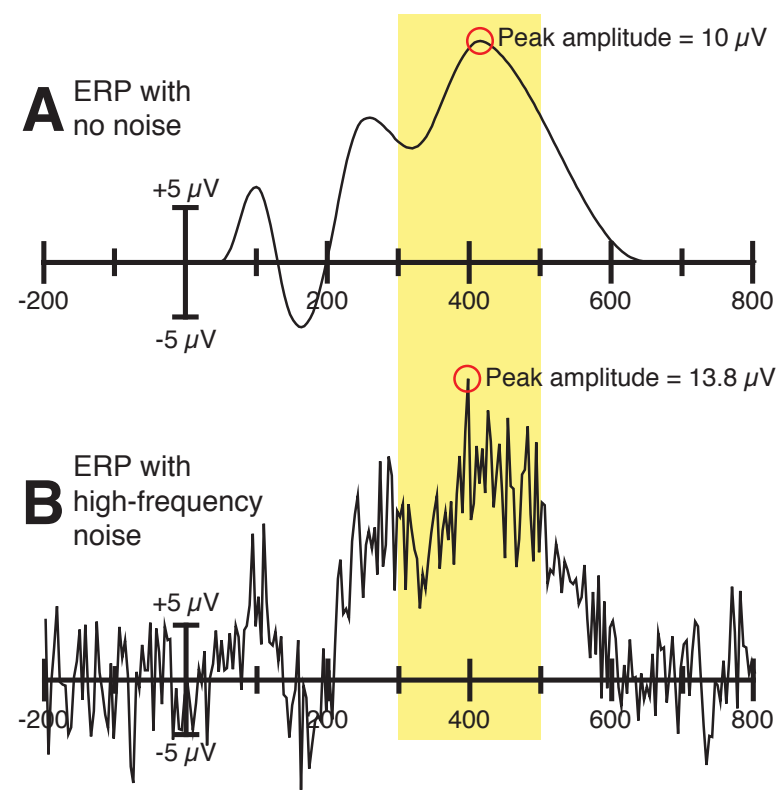

Figure 1. Example ERP waveform without noise (A) and with substantial high-frequency noise (B). High-frequency noise adds significant variability to measurements of peak voltage (indicated by the red circles). However, it has relatively little effect on time-window mean amplitude measures (e.g., the mean voltage from $300-500 \mathrm{~ms}$, shown in yellow shading). amplitude of the P3 wave (or any other component), adding considerable variance and decreasing statistical power. However, this highfrequency noise has much less impact when P3 amplitude is quantified as the mean voltage from 300-500 ms, because the upward and downward deflections largely cancel out (see Clayson, Baldwin, \& Larson, 2013 and Chapter 9 in; Luck, 2014).

One way to quantify the data quality for a specific amplitude or latency measure is to calculate correlation-based measures of reliability (e.g., Boudewyn, Luck, Farrens, \& Kappenman, 2018; Olvet \& Hajcak, 2009). However, this approach provides a single value for an entire group of participants rather than an individual data quality score for each participant. Moreover, these reliability values can be strongly influenced by the range of true scores across individuals, so they do not provide a pure index of data quality (see https://bit.ly/2GTualv for an informal discussion).

Here we propose a universal measure of data quality for ERP research, which we call the standardized measurement error (SME). The SME is closely related to the widely-used

standard error of the mean, but the SME is computed separately for each participant and can be applied to arbitrarily complex ERP measures (e.g., the onset latency of a component that is measured from a difference wave). The SME is designed to answer the question: If you were to obtain an ERP amplitude or latency value in a given participant multiple times in a given paradigm (assuming no learning, fatigue, etc.), how similar would the values be across these 
repeated measurements? As illustrated in Figure B, this is equivalent to quantifying the precision of the amplitude or latency measurement (see Brandmaier et al., 2018).

The SME has a large number of potential uses:

- Determining whether your data quality has increased or decreased when you modify a data analysis step or experimental design feature.

- Indicating technical problems that are reducing your data quality (e.g., degraded electrodes, a poorly trained research assistant).

- Determining whether a given participant's data are too noisy to be included in the analyses or whether a channel is so noisy that it should be replaced with interpolated values.

- Objectively assessing whether the results presented in a published or under-review manuscript are unacceptably noisy.

- Comparing different EEG recording systems, different recording procedures, and different analysis pipelines to see which one yields the best data quality.

The SME is extremely easy to compute when the amplitude of an ERP component is quantified as the mean voltage over a particular time period (which we will call the time-window mean amplitude to distinguish it from a mean across trials). For this simple but common case, we have added functions for calculating the SME to the freely available ERPLAB Toolbox (version
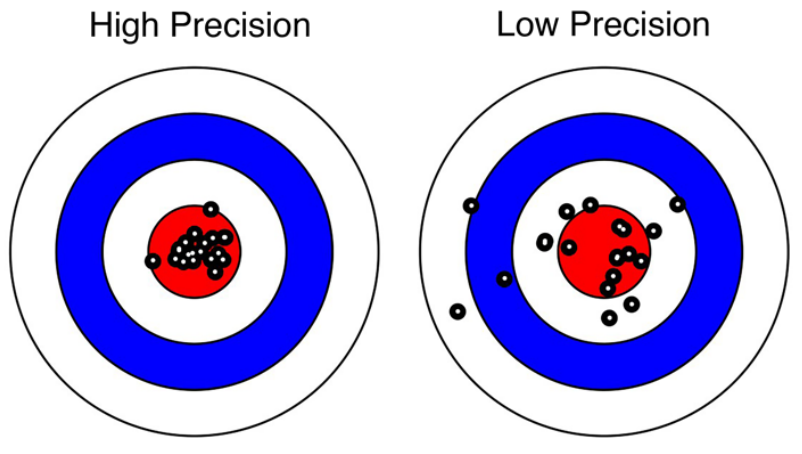

Figure 2. Graphical depiction of the concept of precision. A measure is precise to the extent that it yields the same value every time (assuming that the same thing is being measured). Adapted from Brandmaier et al. (2018) under the terms of a Creative Commons Attribution License (https://creativecommons.org/licenses/by/4.0/).
8 and higher). ERPLAB automatically computes the SME using default time windows (e.g., 100-200 ms, 200-300 ms, etc.) whenever an averaged ERP waveform is created. Custom time windows can also be specified to reflect the time windows used for quantifying component amplitudes in a given experiment.

Computing the SME for other measures (e.g., peak measures, most latency measures) is more complicated and currently requires custom Matlab scripting. However, future versions of ERPLAB Toolbox will provide automated methods for computing the SME for such measures.

\section{THE STANDARD ERROR OF THE MEAN AND DATA QUALITY}

The SME is really just a special application of the standard error of measurement. The standard error of the mean (SEM) is a common case of the standard error of measurement (where the measurement is the mean), so we will first provide a brief review of the SEM. Note that $S M E$ and $S E M$ are quite similar abbreviations, which reflects the fact that they are closely related concepts. 


\subsection{What does the SEM represent?}

The SEM quantifies the expected error in the mean of a set of observations. The SEM is ordinarily computed by taking the standard deviation (SD) of the individual observations and dividing by the square root of the number of observations $(\mathrm{N})$ :

$$
\left.S E M=\frac{S D}{\sqrt{N}} \quad \text { (Equation } 1\right)
$$

As illustrated in Figure 3A, the SEM is widely used in the context of computing the sample mean from a group of participants. For example, if we measured the P3 amplitude in a sample of 20 participants, we could use a bar graph to show the mean across participants and error bars to show the SEM. But what does the SEM actually represent in this context? The SEM answers the question: If we repeated the experiment an infinite number of times, obtaining the mean across participants (the sample mean) every time, how similar would those mean values be? This is analogous to the target example shown in Figure 2: the mean obtained in each replication of the experiment is analogous to a "shot" at the target, and the variation in the sample mean across experiments is analogous to the scatter of these shots. The SEM quantifies the degree of "scatter" in units of SD.
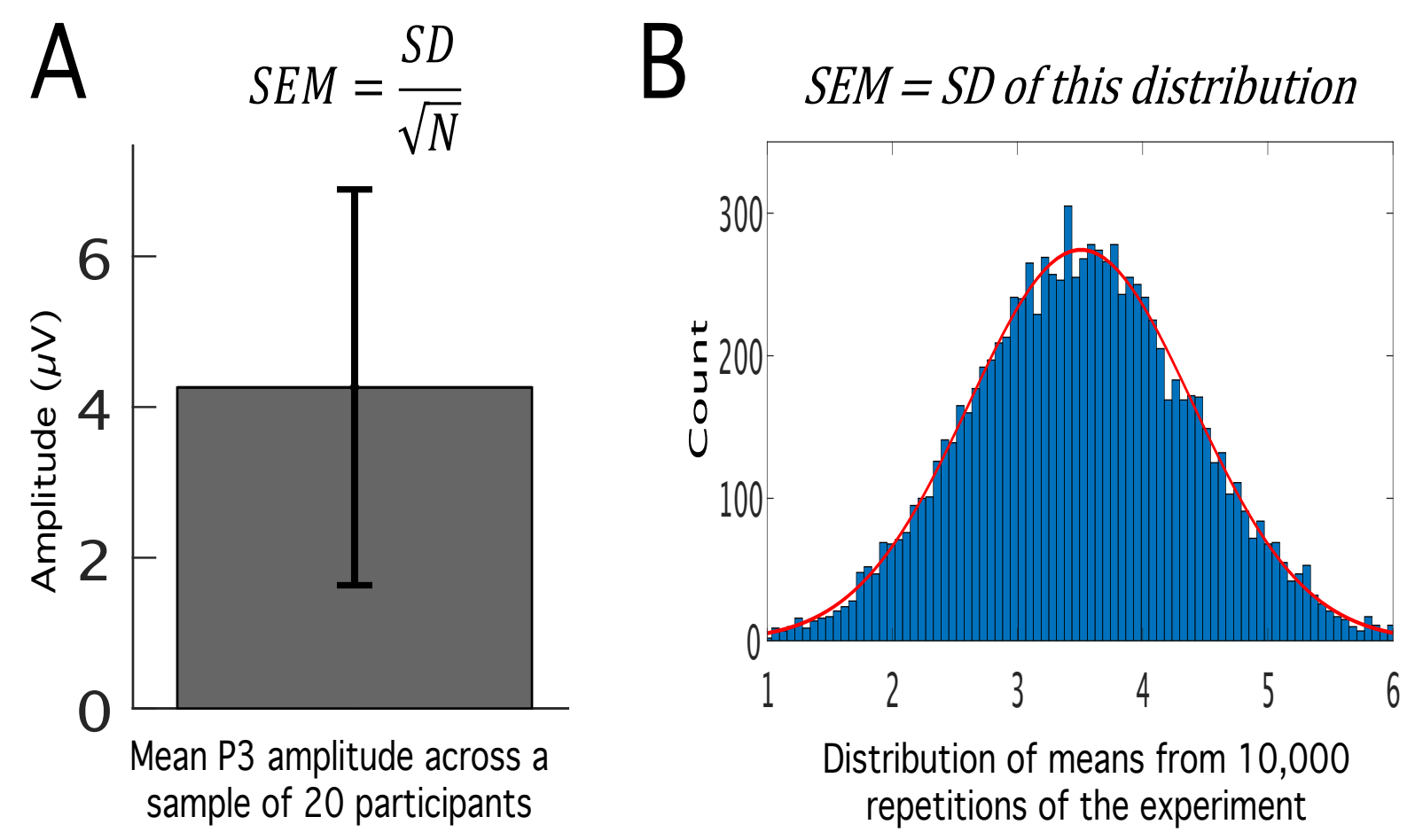

Figure 3. Example of the meaning of the standard error of the mean (SEM). If we obtain a P3 amplitude measure from each of 20 participants, we can calculate the mean of these 20 values and use Equation 1 to compute the SEM. The mean and SEM are often shown as a bar graph with error bars (A). In theory, we could also estimate the SEM by repeating the same experiment a very large number of times. For example, we could repeat the experiment 10,000 times, calculate the mean across the 20 participants for each experiment, and plot a histogram of these values (B). The SD of this distribution is the SEM. 
In theory, we could provide an empirical estimate of the SEM by actually repeating an experiment a large number of times and seeing how much the sample mean varies across experiments. This is illustrated in Figure 3B, which shows the distribution of sample means we might obtain from 10,000 repetitions of an experiment. If we simply take the standard deviation (SD) of this set of sample means, this gives us a very good estimate of the SEM, which is largely equivalent ${ }^{1}$ to the standard SEM formula given in Equation 1. That is, Equation 1 provides a way of using the data from a single experiment to estimate the value we would get by repeating the experiment an infinite number of times, calculating the sample mean each time, and taking the $\mathrm{SD}$ of the sample means. Of course, Equation 1 is much more convenient than repeating the experiment 10,000 times.

When Equation 1 is used to estimate the SEM, we call this the analytic SEM. When the SEM is estimated by repeating the experiment a large number of times, we call this the empirical SEM. Later, we will discuss a third way of estimating the SEM, called the bootstrapped SEM. These are just different ways of estimating the same quantity. The empirical SEM is not actually used in real research because it would be too time-consuming, but it is the best way of understanding what the SEM actually means: The SEM tell us the SD of the observed sample means that we would get by repeating the experiment an infinite number of times.

\subsection{Using the SEM to quantify ERP data quality}

Although the SEM is ordinarily used for a mean across participants, we can instead use it to quantify the expected error in a mean across trials in a single participant. When we use the SEM in this way, it becomes a metric of measurement error (which is what we mean by "data quality," but on a reverse scale because high SEM values indicate low data quality).

Equation 1 can be used to estimate the SEM when we are quantifying the amplitude of an ERP component as the time-window mean amplitude in a single participant's averaged ERP waveform. For example, imagine that we wanted to quantify P3 amplitude as the mean voltage from 300-500 ms, as in Figure 1, from each participant. We will call this measure the participant's score. To obtain the SEM of this score, we would simply measure the mean voltage from 300-500 ms for each of the single-trial EEG epochs, calculate the SD of these single-trial measures, and divide the SD by the square root of the number of trials. This gives us an estimate of the SEM of our score (i.e., the SEM of the mean voltage from 300-500 ms).

If we estimate the SEM in this manner, it answers the question: If we repeated the experiment an infinite number of times with this participant (with no learning, etc.), and we measured the participant's score (the mean amplitude from $300-500 \mathrm{~ms}$ in the averaged ERP waveform) on each repetition, how similar would those scores be? Equation 1 allows us to

\footnotetext{
${ }^{1}$ It is important to distinguish between parameters (e.g., the true SD of a population) and estimates of those parameters (e.g., the SD of a sample, which serves as an estimate of the population SD). For example, when the sample SD is used in Equation 1 instead of the population SD (and the variable is normally distributed), Equation 1 is known to underestimate the true SEM when the number of observations $(\mathrm{N})$ is small $(<\sim 20)$. When the shape of the distribution is unknown, the bias is unknown. However, as long as the number of trials is reasonable (e.g., at least 10), and the distribution isn't too different from normal, the bias should be modest. Note that, to avoid complex notation in this brief paper, we have simply used the term "estimated" before the name of a parameter when the statement refers only to an estimate of the parameter and not to the parameter itself.
} 
estimate the answer to this question - using units of SD-without repeating the experiment multiple times.

You can also think of the SEM as quantifying your uncertainty about the true value of the participant's score. The smaller the SEM, the more certain you are that the score you actually measured from the participant's averaged ERP waveform (in a single experiment) is close to the value you would get by repeating that experiment an infinite number of times and averaging the scores across the experiments (assuming no learning, fatigue, etc.). Conversely, a large SEM means that you have little confidence that the score you measured from this participant is close to the participant's true score. In other words, it provides a metric of measurement error.

When the SEM is used to quantify single-participant data quality in this manner, we call it the standardized measurement error or SME because it is a metric of measurement error that uses standardized units (i.e., units of SD). In this particular case, the SME is simply the estimated SEM computed via Equation 1 from single-trial measurements of the time-window mean amplitude. However, this is just a special case of the SME, and the SME can also be used for other ERP scores, such as latency measures. In fact, the SME can even be used when a score is obtained from a difference wave or some other transformation of the data. Unfortunately, Equation 1 cannot be used to estimate the SME in these cases, but the next section will describe an alternative means of estimating the SME that works with virtually any measure that can be obtained from an averaged ERP waveform.

Before moving on to this alternative approach, we would like to make it explicit that the SME satisfies our two main criteria for a universal measure of data quality: 1) it provides a separate metric of data quality for each individual participant, and 2) it reflects the quality of the actual score that we will be putting into our statistical analysis (e.g., the mean voltage from 300$500 \mathrm{~ms}$, measured from the averaged ERP waveform). A later section will describe how the SME values can be aggregated across participants to reflect the data quality in a group of participants.

Here is a more formal definition of the SME in the context of ERP research: The standardized measurement error (SME) is the standard error of measurement for the amplitude or latency of an ERP component measured from a single-participant ERP waveform.

Because the SME quantifies the measurement error for a particular amplitude or latency score, any source of trial-to-trial variability that influences that score is considered a source of error. For example, alpha-band EEG oscillations can add trial-to-trial variability when the amplitude of the P3 wave is scored as the peak amplitude, these oscillations are considered a source of measurement error for this score. In a different study, however, these same alpha-band oscillations could be the signal of interest rather than a source of measurement error. Thus, one can have high measurement error (and a large SME value) as a result of bona fide neural signals, and data that produce poor data quality for one analysis might produce excellent data quality for a different analysis.

\section{GENERALIZATION TO OTHER ERP AMPLITUDE AND LATENCY SCORES}

The analytic SEM formula (Equation 1) can be used when our score is the time-window mean amplitude measure (e.g., the mean voltage from 300-500 ms), but it cannot be validly applied to most other methods for scoring ERP amplitudes and latencies (e.g., peak amplitude or peak latency). It is valid for the time-window mean amplitude because the score obtained from the averaged ERP waveform is exactly the same as what we would get by obtaining this score on 
each individual trial and then averaging those single-trial scores together (see Figure 4). Because we get exactly the same value for the time-window mean amplitude whether we measure from the single-trial epochs or from the averaged ERP waveform, the SEM estimated from the singletrial scores using Equation 1 is also the estimated SEM of the score obtained from the averaged ERP waveform. That is, it is the same score, so it has the same SEM.

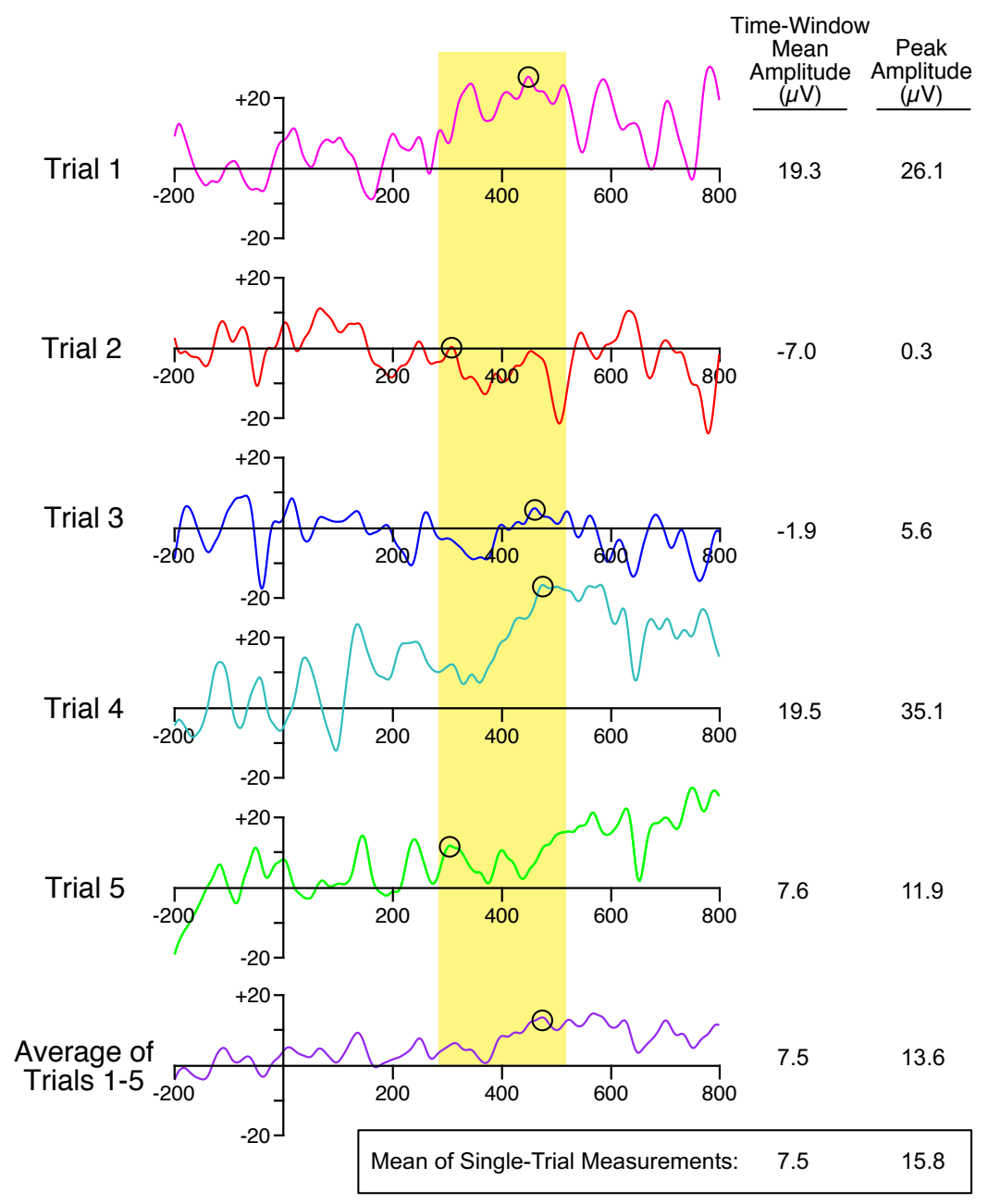

Figure 4. Five single-trial EEG epochs, the average of these epochs, and the time-average and peak amplitude measures from each epoch and from the averaged waveform. The mean of the timewindow mean amplitudes from the five individual trials is identical to the time-window mean amplitude measured from the averaged waveform. However, the mean of the peak amplitudes of the five EEG epochs is not the same as the peak amplitude of the averaged waveform.

Now consider what would happen if we instead used the peak amplitude between 300 and $600 \mathrm{~ms}$ as our score. If we measured this score from the individual trials and then averaged those scores together, the result would not be the same as measuring the peak amplitude from the averaged ERP waveform (see Figure 4). Because the average of the single-trial scores is not the same as the score obtained from the averaged ERP waveform, the SEM of the single-trial peak amplitude scores is not the standard error of the peak amplitude score obtained from the averaged ERP waveform. These are different scores, so they have different standard errors, and 
Equation 1 does not give us the standard error of the peak amplitude measured from the averaged ERP waveform.

This problem applies to almost every ERP amplitude or latency measure with the exception of the time-window mean amplitude (including peak amplitude, peak latency, and every current measure of onset or offset latency). Could we then just use the mean of the singletrial scores (for which Equation 1 can be used to estimate the SEM) as our dependent variable? Unfortunately, the answer is usually "no" for these measures, because they would be so distorted by the single-trial noise that the average of the single-trial scores will typically be useless as a dependent variable. Consequently, we must obtain the scores from the averaged ERP waveforms in most cases, so we need a different method for obtaining the standard errors for these scores.

Fortunately, we can use a method called bootstrapping to estimate the standard error for the peak amplitude, the peak latency, or virtually any score that can be obtained from an averaged ERP waveform. Bootstrapping is based on a simple but powerful idea: We can simulate a new experiment from an existing set of $\mathrm{N}$ trials by randomly sampling $\mathrm{N}$ trials from the existing data set with replacement. Because we sample with replacement, we get a different set of $\mathrm{N}$ trials and therefore a different averaged ERP waveform every time we simulate an experiment.

This allows us to simulate the empirical procedure for estimating the standard error shown in Figure 3B. For each simulated experiment, we sample N trials with replacement from the set of $\mathrm{N}$ trials for a given condition of that experiment, create an averaged ERP waveform for that condition, and obtain the score of interest from this averaged ERP waveform (e.g., P3 peak latency). We can then compute the estimated SD of the scores obtained across many simulated experiments. Because the standard error is defined as the SD of the scores across a large number of repetitions, the SD we compute in this manner is an estimate of the standard error of measurement for our ERP score.

For example, imagine that we conduct an oddball experiment in which each participant receives 20 oddball trials and 80 standard trials, and we are interested in the peak latency of the P3 wave (i.e., the time at which the voltage reaches the maximum value between 300 and 800 $\mathrm{ms})$. Our score in this experiment is the peak latency, and the standardized measurement error (SME) for this score is simply the standard error of measurement for each of our two trial types in a given participant.

To compute the estimated SME for the oddballs, we would randomly sample 20 trials with replacement from the 20 oddball trials we actually collected from this participant. We would then make an averaged ERP waveform from these 20 trials and find the peak latency from this averaged waveform. We would then repeat this process many times (e.g., 10,000 times), getting a new random sample of trials on each iteration. This would give us 10,000 peak latency scores, and our estimate of the SME for the oddball trials would be the SD of these 10,000 scores.

We would do the same thing for the standard trials, except that we would randomly sample (with replacement) 80 trials from the set of 80 standard trials. These averages will have less noise than the averages from the oddball trials because more trials are being averaged together. Consequently, we would expect the SD of the 10,000 peak latency scores to be smaller for the standards than for the oddballs. This is very sensible: We are using the SME to quantify 
the quality of the data obtained from the averaged ERP waveforms, so the SME should be better (smaller) when the waveforms are based on more trials (all else being equal).

When we estimate the SME using the analytic SEM formula shown in Equation 1, we call this the analytic SME. When we estimate the SME using bootstrapping, we call it the bootstrapped SME. We can use either the analytic SME or the bootstrapped SME when our score is the time-window mean amplitude, but the analytic SME is much faster and more convenient to compute. For most other scores, we must use bootstrapping to estimate the $\mathrm{SME}^{2}$.

\section{AGGREGATING SME VALUES ACROSS PARTICIPANTS}

A key virtue of the SME is that it is obtained from individual participants, so it can be used to identify participants with noisy data or even noisy channels within individual participants. However, it can also be useful to provide a single SME value that is aggregated across participants to represent the of data quality of the entire data set. For example, it would be very appropriate for a published ERP paper to provide an aggregate SME value for each condition of the study so that the data quality could be compared with other similar studies.

One could simply use the mean of the single-participant SME scores in each condition as the aggregate value. However, there is a different way of aggregating the values that is more directly related to the effect of the measurement error on statistical power. It turns out that participants with higher-than-average measurement error have an outsized deleterious impact on the expected effect size of a given experiment, and this alternative way of aggregating the data takes this into account.

Specifically, because each SME value is in SD units, squaring the values puts them into units of variance, which makes it straightforward to combine the values across participants (because the variance of the sum of a set of independent random variables is equal to the sum of the individual variances). In particular, if the squared values are averaged together, this provides an estimate of the portion of the variance in the original scores (e.g., the P3 latency scores) that is attributable to measurement error, which in turn is directly related to the effect size and the statistical power. However, the average of the squared values is not in the same units as the original score. Thus, it is convenient to take the square root of the average of the squared SME scores. This is the root mean square (RMS) of the SME or RMS(SME). RMS(SME) is our preferred aggregate measure of data quality (although the mean of the single-participant SME values is also acceptable).

Aggregating across participant by means of the RMS makes it possible to estimate how your effect sizes and statistical power would change if you increased or decreased the number of trials in an experiment. The total variance observed in a given experiment can be broken into true score variance (true differences across individuals that would be present even with an infinite number of trials per participant) and measurement error variance. If you square the estimated RMS(SME) value, this provides an estimate of the measurement error variance. Increasing or decreasing the number of trials per participant will change the measurement error

\footnotetext{
${ }^{2}$ Because bootstrapping uses the actual samples as a proxy for the true distribution of values, it works better (in terms of bias and precision) for larger samples sizes than for smaller samples. However, bootstrapping works surprisingly well for modest sample sizes. In preliminary applications to real data sets, we have found very sensible analytic and bootstrapped SME results with 20 trials per condition.
} 
variance in a very predictable way, because the measurement error variance is linearly related to the number of trials (all else being equal).

If you subtract the measurement error variance from the observed total variance, the result will be an estimate of the true score variance, which does not vary as a function of the number of trials (assuming no learning, etc.). Baker et al. (2019) have provided a power calculator that allows you to use the true score variance and measurement error variance to estimate how your statistical power will change as a function of the number of trials in the experiment.

Note that ERPLAB Toolbox makes it easy to aggregate the values across participants when a grand average ERP waveform is being computed. The user can choose whether to aggregate by computing RMS(SME) or by simply averaging the single-participant SME values.

Thus, not only does the SME provide a single-participant estimate of data quality that pertains to the specific score being used as the dependent variable in a given experiment, the estimated SME values can be aggregated across participants using RMS to aid in power calculations. 


\section{References}

Baker, D. H., Vilidaite, G., Lygo, F. A., Smith, A. K., Flack, T. R., Gouws, A. D., \& Andrews, T. J. (2019). Power contours: Optimising sample size and precision in experimental psychology and human neuroscience. ArXiv:1902.06122 [q-Bio, Stat]. Retrieved from http://arxiv.org/abs/1902.06122

Boudewyn, M. A., Luck, S. J., Farrens, J. L., \& Kappenman, E. S. (2018). How Many Trials Does It Take to Get a Significant ERP Effect? It Depends. Psychophysiology, 55, e13049.

Brandmaier, A. M., Wenger, E., Bodammer, N. C., Kühn, S., Raz, N., \& Lindenberger, U. (2018). Assessing reliability in neuroimaging research through intra-class effect decomposition (ICED). ELife, 7. https://doi.org/10.7554/eLife.35718

Clayson, P. E., Baldwin, S. A., \& Larson, M. J. (2013). How does noise affect amplitude and latency measurement of event-related potentials (ERPs)? A methodological critique and simulation study. Psychophysiology, 50, 174-186.

Luck, S. J. (2014). An Introduction to the Event-Related Potential Technique, Second Edition. Cambridge, MA: MIT Press.

Olvet, D. M., \& Hajcak, G. (2009). Reliability of error-related brain activity. Brain Research, 1284, 89-99. 